Revista Brasileira de Agricultura Irrigada v.14, nº.1, p. 3792 - 3802, 2020

ISSN 1982-7679 (On-line)

Fortaleza, CE, INOVAGRI - http://www.inovagri.org.br

DOI: $10.7127 /$ rbai.v14n100955

Protocolo 955.20 - 30/05/2018 Aprovado em 26/05/2020

\title{
CRESCIMENTO E RENDIMENTO DA CANA-DE-AÇÚCAR IRRIGADA SUBMETIDA A DIFERENTES REPOSIÇÕES HÍDRICAS E NITROGÊNIO
}

\author{
Nelmício Furtado da Silva ${ }^{1}$, Fernando Nobre Cunha ${ }^{2}$, Marconi Batista Teixeira ${ }^{3}$, Frederico Antonio \\ Loureiro Soares ${ }^{3}$
}

\begin{abstract}
RESUMO
A cultura da cana-de-açúcar é a principal matéria-prima usada na fabricação de açúcar e álcool. A melhoria na sua qualidade pode ser obtida através do uso da irrigação e da adubação nitrogenada, que também promove aumento de sua produtividade. Dessa forma, objetivou-se com este estudo avaliar o desenvolvimento da cana-de-açúcar nas diferentes fases de cultivo e o rendimento final de álcool e açúcar em função da adubação nitrogenada com diferentes reposições hídricas. O delineamento experimental utilizado foi o de blocos ao acaso, em esquema fatorial $5 \times 2 \times 2$, com quatro repetições. Os tratamentos consistiram em cinco níveis de reposição hídrica $(100,75,50,25$ e $0 \%$, com base na umidade do solo na capacidade de campo), combinados com e sem aplicação de fertilizante nitrogenado ( 0 e $100 \mathrm{~kg} \mathrm{ha}^{-1}$ de $\mathrm{N}$ ) na forma de ureia, em dois ciclos de cultivo (canaplanta e cana-soca). O aumento da reposição hídrica combinada com adubação nitrogenada proporciona incrementos de aproximadamente $10 \%$ no número de entrenós da cana-de-açúcar. A reposição hídrica combinada com a adubação nitrogenada promoveu aumento linear do rendimento bruto de açúcar e de álcool.
\end{abstract}

Palavras-chave: Ciclo de cultivo, irrigação subsuperficial, adubação nitrogenada, álcool, açúcar.

\section{GROWTH AND YIELD OF IRRIGATED SUGARCANE SUBMITTED TO DIFFERENT WATER REPLACEMENTS AND NITROGEN}

\begin{abstract}
The sugarcane crop is considered the main source of raw material for sugar and alcohol manufacture. The improvement in its quality can be applied through the use of irrigation and nitrogen fertilization, which also promoted an increase of its yield. Thus, the objective of this study was to evaluate the

\footnotetext{
${ }^{1}$ Eng. Agrônomo, Doutor em Ciências Agrárias - Agronomia, IF Goiano - Campus Rio Verde, Rio Verde - GO, Rodovia Sul Goiana, Km 01, CEP: 75.901-170, Rio Verde-GO, e-mail: nelmiciofurtado@gmail.com

${ }^{2}$ Eng. Agrônomo, Doutor em Ciências Agrárias - Agronomia, Instituto Federal Goiano - Campus Rio Verde, Rio Verde - GO, e-mail: fernandonobrecunha@hotmail.com

${ }^{3}$ Eng. Agrônomo, Prof. Dr. em Agronomia, IF Goiano - Campus Rio Verde, Rio Verde - GO, Rio Verde - GO, e-mail: marconibt@gmail.com
} 
development of sugarcane in the various stages of cultivation and the final yield of alcohol and sugar in a function of nitrogen fertilization with different water replenishments. The experimental design was a randomized block in a factorial scheme $5 \times 2 \times 2$, with four replications. The treatments consisted of five levels of fluid replacement $(100,75,50,25$ and $0 \%$, based on soil moisture at field capacity) combined with and without application of nitrogen fertilizer $\left(0\right.$ and $\left.100 \mathrm{~kg} \mathrm{ha}^{-1} \mathrm{~N}\right)$ in the form of urea in two cycles of cultivation (plant and ratoon crops). The increase in water replacement combined with nitrogen fertilization provides an increase of approximately $10 \%$ in the number of sugarcane internodes. Water replacement combined with nitrogen fertilization promoted a linear increase in the gross sugar and alcohol yield.

Keywords: cultivation cycle, subsurface irrigation, nitrogen fertilization, alcohol, sugar.

\section{INTRODUÇÃO}

A cultura da cana-de-açúcar é considerada a principal fonte de matéria-prima para fabricação de açúcar e álcool (etanol). Os colmos, parte da planta de maior importância econômica, podem ser divididos basicamente nos seguintes constituintes de interesse agronômico: a fibra (10 a 12\%), composta por celulose, hemicelulose e lignina; e o caldo (82 a $90 \%$ ), que apresenta 75 a $82 \%$ de água e 18 a $25 \%$ de sólidos solúveis (estádio de maturação), sendo este o constituinte de maior importância na indústria sucroalcooleira. Dos sólidos solúveis presentes no caldo, 1 a $2 \%$ são não açúcares (sais inorgânicos e orgânicos) e o restante composto por açúcares, sendo 14 a $24 \%$ sacarose, 0,2 a $1,0 \%$ glicose e 0,0 a $0,5 \%$ frutose (MASCHIO, 2011).

$\mathrm{Na}$ cana-planta, o ciclo evolutivo da cultura pode ser de 12 meses (cana de ano) e 18 meses (cana de ano e meio). Após o primeiro corte o ciclo passa a ser de 12 meses, e a partir do primeiro corte passa a ser denominada de cana-soca (BARBOSA, 2010). Porém, existem diversas variedades de ciclo precoce, que permitem uma maior flexibilidade no planejamento do plantio e da colheita.

As principais características morfológicas deste gênero são o crescimento do caule em colmos, as folhas que possuem lâminas de sílica nas suas bordas e as bainhas abertas. O hábito de crescimento da cana-deaçúcar pode variar de ereto, semi-decumbente a decumbente, de acordo com a característica da variedade. Esta cultura apresenta sistema fotossintético $\mathrm{C} 4$, que segundo Taiz e Zeiger
(2004), em maiores temperaturas $\left(30\right.$ a $\left.40{ }^{\circ} \mathrm{C}\right)$, possuem alto desempenho do metabolismo de fotossíntese. Vale destacar que este desempenho é afetado por outros fatores do clima, pelos parâmetros físico-químicos dos solos, manejo e práticas culturais e, de maneira fundamental, pela disponibilidade de água no solo (MASCHIO, 2011).

Esses fatores que interferem na produção e na qualidade da cana-de-açúcar, bem como de seus subprodutos, foram sendo constantemente estudados sob diferentes aspectos (OLIVEIRA et al., 2007). A disponibilidade de água para a cana-de-açúcar é o principal fator climático causador de variabilidade da produtividade. Entretanto, devido às variações locais de clima e de variedades, é difícil estabelecer uma relação entre produção e consumo de água pela canade-açúcar (GOUVÊA, 2008).

A melhoria da qualidade da cana-deaçúcar pode ser obtida com o uso da irrigação (GOUVEIA NETO, 2012). A irrigação, além de aumentar a produtividade da cana-deaçúcar, promove melhorias nos índices de qualidade da matéria-prima (FARIAS et al., 2009).

Vários fatores podem interferir na qualidade final da matéria prima. $\mathrm{O}$ rendimento e a produção de açúcar e de álcool da cana-de-açúcar irrigada dependem da quantidade de água aplicada, do manejo de irrigação combinado com a quantidade certa de adubação, da variedade, da idade do corte, do tipo de solo e do clima (DANTAS NETO et al., 2006).

Diante do exposto, objetivou-se com este estudo avaliar o desenvolvimento da cana- 


\section{CRESCIMENTO E RENDIMENTO DA CANA-DE-AÇÚCAR IRRIGADA SUBMETIDA A DIFERENTES REPOSIÇÕES HÍDRIÇAS E NITROGÊNIO}

de-açúcar, o rendimento final de álcool e de açúcar em diferentes fases de cultivo e em dois ciclos em função da adubação nitrogenada com diferentes reposições hídricas, via sistema de gotejamento subsuperficial.

\section{MATERIAL E MÉTODOS}

O experimento foi conduzido na Área Experimental do Instituto Federal Goiano Campus Rio Verde-GO, localizada na latitude $17^{\circ} 48^{\prime} 28^{\prime \prime S}$ e longitude 5053'57" O, com altitude média de $720 \mathrm{~m}$ e relevo suave ondulado ( $6 \%$ de declividade).
O clima da região é do tipo Aw (tropical), conforme Köppen e Geiger (1928), com as precipitações concentradas nos meses de outubro a maio e com estiagem nos meses de junho a setembro.

A temperatura anual varia de 20 a $35^{\circ} \mathrm{C}$ e as precipitações variam de 1.500 a $1.800 \mathrm{~mm}$ anuais. $\mathrm{O}$ solo foi classificado como Latossolo Vermelho distroférrico (LVdf) de textura média (EMBRAPA, 2013). Na Tabela 1 estão descritas as características físicohídricas e químicas do solo, observadas antes da implantação do experimento.

Tabela 1. Características físico-hídricas e químicas, nas camadas de $0-0,20$ e $0,20-0,40 \mathrm{~m}$ de profundidade de um Latossolo Vermelho distroférrico.

\begin{tabular}{|c|c|c|c|c|c|c|c|c|c|c|c|}
\hline \multicolumn{12}{|c|}{ Características físico-hídricas } \\
\hline \multirow{2}{*}{$\begin{array}{c}\text { Camada } \\
\text { (m) }\end{array}$} & \multicolumn{3}{|c|}{ Granulometria $\left(\mathrm{g} \mathrm{kg}^{-1}\right)$} & $\theta_{\mathrm{CC}}$ & $\theta_{\mathrm{PMP}}$ & Ds & \multirow{2}{*}{\multicolumn{2}{|c|}{$\begin{array}{c}\text { PT } \\
\mathrm{cm}^{3} \mathrm{~cm}^{-3}\end{array}$}} & \multirow{2}{*}{\multicolumn{3}{|c|}{ Classificação textural }} \\
\hline & Areia & Silte & Argila & \multicolumn{2}{|c|}{$---\mathrm{m}^{3} \mathrm{~m}^{-3}---$} & $\mathrm{g} \mathrm{cm}^{-3}$ & & & & & \\
\hline $0-0,20$ & 458,3 & 150,2 & 391,5 & 51,83 & 30,5 & 1,27 & & 0,55 & \multicolumn{3}{|c|}{ Franco Argiloso } \\
\hline $0,20-0,40$ & 374,9 & 158,3 & 466,8 & 55 & 31,33 & 1,28 & & 0,51 & & Argila & \\
\hline \multicolumn{12}{|c|}{ Características químicas } \\
\hline Camada & $\mathrm{pH}$ & MO & $\mathrm{P}$ & K & $\mathrm{Ca}$ & $\mathrm{Mg}$ & $\mathrm{Al}$ & $\mathrm{H}+\mathrm{Al}$ & $S$ & CTC & $\mathrm{V}$ \\
\hline (m) & em $\mathrm{H}_{2} \mathrm{O}$ & $\mathrm{g} \mathrm{kg}^{-1}$ & $\mathrm{mg} \mathrm{dm}{ }^{-3}$ & \multicolumn{7}{|c|}{-- } & $(\%)$ \\
\hline $0,0-0,20$ & 6,2 & 63,42 & 7,06 & 2,04 & 20,40 & 16,80 & 0,0 & 57,75 & 41,80 & 99,55 & 41,99 \\
\hline $0,20-0,40$ & 6,6 & 44,47 & 2,65 & 4,09 & 14,40 & 13,20 & 0,0 & 44,55 & 31,69 & 76,24 & 41,57 \\
\hline
\end{tabular}

$\theta_{\mathrm{CC}}$, capacidade de campo $(10 \mathrm{KPa})$; $\theta_{\mathrm{PMP}}$, ponto de murcha permanente $(1.500 \mathrm{KPa})$; Ds, densidade do solo; PT, porosidade total; $\mathrm{pH}$ em água destilada. P e K, extrator Mehlich ${ }^{-1}$. M.O - Matéria orgânica. V - Saturação por bases.

O delineamento experimental utilizado foi o de blocos ao acaso, em esquema fatorial $5 \times 2 \times 2$, com quatro repetições. Os tratamentos consistiram em cinco níveis de reposição hídrica - $\mathrm{RH}(100,75,50,25$ e $0 \%$, com base na umidade do solo na capacidade de campo), combinados com e sem aplicação de fertilizante nitrogenado - N (0 e $100 \mathrm{~kg} \mathrm{ha}^{-1}$ de $\mathrm{N}$ ) na forma de ureia, em dois ciclos de cultivo- $\mathrm{C}$ (cana-planta e cana-soca).

Para a condução do ensaio, selecionouse uma área de $2.300 \mathrm{~m}^{2}$, que antes do plantio da cana-de-açúcar, foi cultivada por 20 anos com pastagem. O preparo inicial do solo consistiu de gradagem prévia com o intuito de eliminar a vegetação existente, além da distribuição de calcário dolomítico na dosagem de 2,0 tha ${ }^{-1}$, com intuito de elevar a saturação por bases, conforme recomendado por Sousa e Lobato (2004). O corretivo foi distribuído por meio de distribuidora de calcário tratorizada, e posteriormente realizouse outra gradagem com o propósito de incorporar o calcário e destorroar o solo. Por 
último, realizou-se a gradagem de nivelamento.

O plantio da cana-de-açúcar foi realizado em março de 2011, utilizando-se a variedade RB 85-5453, que apresenta como características principais, alto teor de açúcar e precocidade. As 80 parcelas experimentais foram compostas por três sulcos de linha dupla (plantio "em W") com espaçamento de 1,4 m entre fileiras duplas x $0,4 \mathrm{~m}$ entre linhas e $8 \mathrm{~m}$ de comprimento, totalizando $43,2 \mathrm{~m}^{2}$ de área total por parcela.

Nos tratamentos com reposição hídrica, foi utilizado o método de irrigação por gotejamento subsuperficial. O tubo gotejador (modelo Dripnet PC 16150 com parede delgada) foi enterrado a $0,20 \mathrm{~m}$ de profundidade, no meio da linha dupla. O sistema operava com pressão de serviço de 1,0 bar, vazão nominal de $1,0 \mathrm{~L} \mathrm{~h}^{-1}$ e espaçamento entre gotejadores de $0,50 \mathrm{~m}$.

A irrigação foi conduzida com base em tensiometria digital de punção, com sensibilidade de $0,1 \mathrm{kPa}$, sendo as hastes tensiométricas instaladas nas profundidades de 0,20 e 0,40 e distâncias de $0,15,0,30,0,45$ e $0,60 \mathrm{~m}$ do tubo gotejador, com leitura do potencial matricial do solo $(\Psi \mathrm{m})$ registrada diariamente. Para determinar a necessidade de irrigação, utilizou-se tensão crítica de $40 \mathrm{kPa}$, na profundidade de $0,20 \mathrm{~cm}$.

As características físico-hídricas do solo foram determinadas obtendo-se, assim, a curva de retenção de água no solo, a partir do uso do software RETEC (VAN GENUCHTEN, 2009), que possibilita gerar os parâmetros empíricos de ajuste da equação de van Genuchten (1980), convertendo o $\Psi \mathrm{m}$ mensurado em campo em conteúdo de água no solo $(\theta)$, conforme a equação de van Genutchen (1980) (Equação 1). $\theta=\theta_{r}+\frac{\theta_{s}-\theta_{r}}{\left[1+\left(\alpha \times\left|\psi_{m}\right|\right)^{n}\right]^{m}}$

Em que: $\theta$ - conteúdo de água no solo, $\mathrm{cm}^{3} \mathrm{~cm}^{-3} ; \Psi_{\mathrm{m}}-$ potencial mátrico, $\mathrm{kPa} ; \theta_{\mathrm{s}}-$ umidade do solo saturado, $\mathrm{cm}^{3} \mathrm{~cm}^{-3} ; \theta_{r}-$ umidade do solo residual, $\mathrm{cm}^{3} \mathrm{~cm}^{-3} ; \alpha, \mathrm{n}, \mathrm{m}-$ parâmetros de ajuste empíricos.

Os resultados diários do conteúdo de água no solo foram utilizados para determinar o volume de água aplicado para cada reposição hídrica, sendo que nos tratamentos de $100 \%$ baseou-se na elevação da umidade do solo até a capacidade de campo. Para os demais tratamentos, foram aplicadas lâminas de acordo com a porcentagem prevista de reposição hídrica.

Todas as parcelas foram adubadas nos sulcos de plantio, conforme o resultado da análise de solo e recomendação de Sousa e Lobato (2004), com aplicação de $120 \mathrm{~kg} \mathrm{ha}^{-1}$ de $\mathrm{P}_{2} \mathrm{O}_{5}$ (superfosfato simples) e $80 \mathrm{~kg} \mathrm{ha}^{-1} \mathrm{de}$ $\mathrm{K}_{2} \mathrm{O}$ (cloreto de potássio). $\mathrm{Na}$ condição de cana-planta e cana-soca, nas parcelas em que foi prevista a aplicação de $100 \mathrm{~kg} \mathrm{ha}^{-1}$ de $\mathrm{N}$ (ureia), este foi aplicado totalmente via água de irrigação (fertirrigação), parcelado em dez aplicações ao longo do ciclo da cultura. Todas as parcelas experimentais continham sistema de irrigação para aplicação da fertirrigação.

A partir dos dados climatológicos do período experimental, foi elaborada a estimativa do balanço hídrico decendial para a canade-açúcar em manejo de sequeiro, empregando-se o método de Thornthwaite e Mather (1955), sendo que a evapotranspiração de referência $\left(\mathrm{ET}_{0}\right)$ foi calculada segundo a equação de Penman-Monteith-FAO/56 (ALLEN et al., 1998) (Figura 1). 

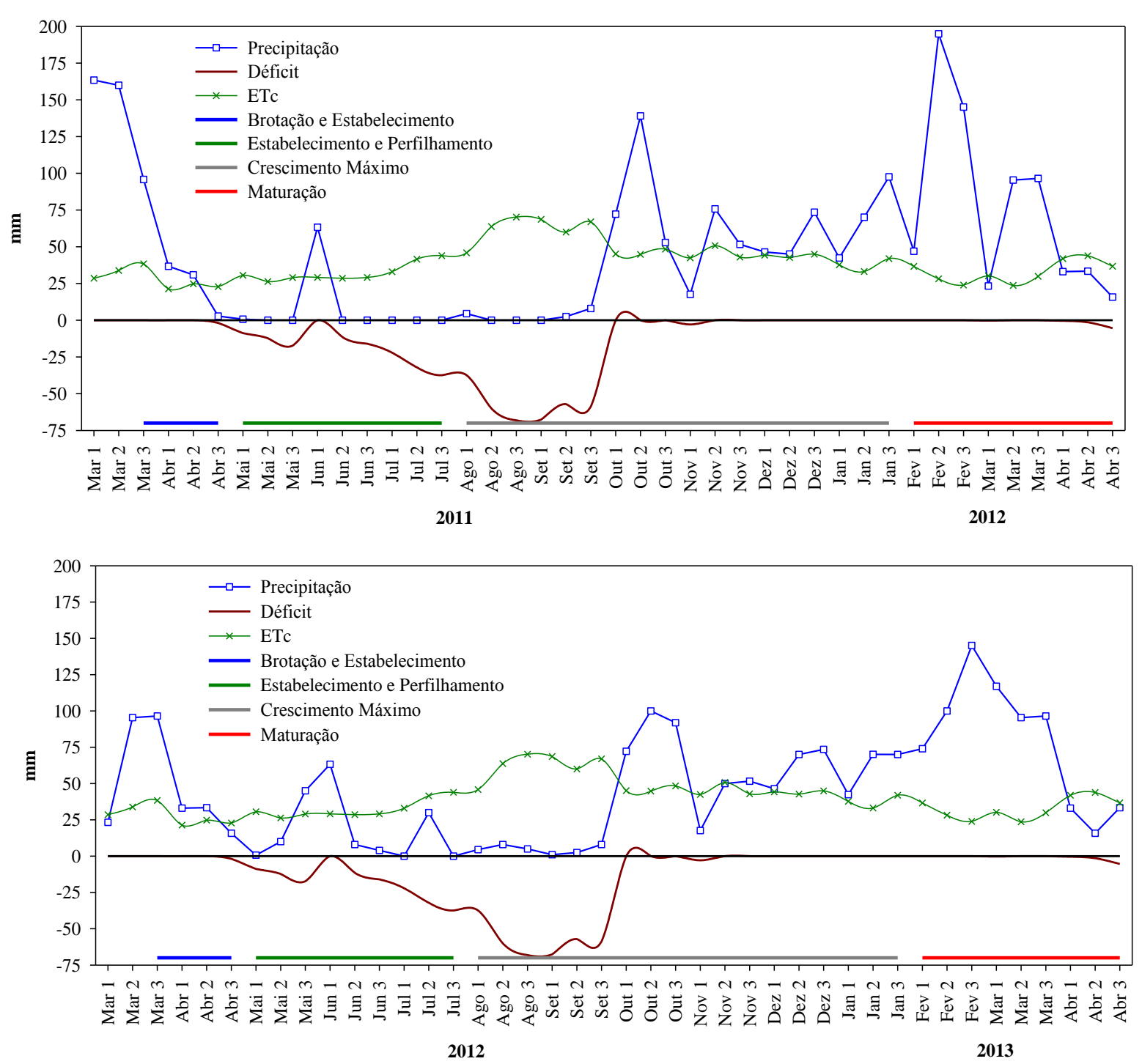

Fonte: Estação Normal INMET - Rio Verde - GO.

Figura 1. Balanço hídrico da cana-de-açúcar em manejo de sequeiro. DEF - Déficit hídrico. ETc Evapotranspiração da cultura. Fases da cultura (adaptado de Doorenbos e Kassam, 1994): Brotação e Estabelecimento $(\mathrm{Kc}=0,6)$; Estabelecimento e Perfilhamento $(\mathrm{Kc}=0,9$ a 1,1$)$; Crescimento Máximo $(\mathrm{Kc}=$ $1,3)$; e Maturação ( $\mathrm{Kc}=0,7$ a 0,9$)$.

O número de entrenós (NE) foi obtido pela contagem em todos os colmos das plantas a partir do destacamento dos primeiros colmos. Foram demarcadas três plantas aleatórias em um metro linear no centro da linha dupla principal, para análise mensal da variável.

Depois das avaliações, os dados foram agrupados segundo os estádios de desenvolvimento da cana-de-açúcar. Conforme Diola e Santos (2012), os estádios de desenvolvimento da cana-de-açúcar se dividem em quatro fases, a saber: (a) Brotação e estabelecimento da cultura; (b) Perfilhamento: estende-se desde o final da brotação até 120 dias após o plantio; (c) Desenvolvimento vegetativo e crescimento dos colmos: inicia-se logo após a fase de perfilhamento até 270 dias após o plantio; (d) Maturação: fase de síntese e acúmulo de açúcar, que dura de 270-300 até 360 dias após o plantio.Sendo que neste estudo as avaliações iniciaram após o estádio Brotação e estabelecimento da cultura (a), além disso, 
cada estádio foi chamado de fase: (b) Fase I perfilhamento; (c) Fase II - crescimento máximo; (d) Fase III - maturação.

Foi realizado o monitoramento dos sólidos solúveis ( ${ }^{\circ}$ Brix) da cana-de-açúcar em campo, nas três últimas semanas antes da colheita em cada ciclo de cultivo. Para a determinação racional do ponto de colheita da cana-de-açúcar, utilizou-se o parâmetro conhecido como Índice de Maturação (IM) determinado em campo, utilizando-se um refratômetro portátil.

Os valores de IM são: (a) menor que 0,60 para cana verde; (b) entre 0,60 e 0,85 para cana em processo de maturação; (c) entre 0,85 e 1 para cana madura; e (d) maior que 1 para cana em processo de declínio de sacarose (ROSSETTO, 2006). No momento em que se atingiu Índice de Maturação (IM $={ }^{\circ}$ Brix do Ápice $/{ }^{\circ}$ Brix da Base) entre 0,9 e 0,95 , foi realizada a colheita.

A produtividade de colmos foi determinada através da pesagem total dos colmos presentes na área útil das respectivas parcelas sendo quantificado o peso dos colmos presentes nas duas linhas centrais de cada parcela, cujo valor foi extrapolado para $\mathrm{tha}^{-1}$. Para tanto, realizou-se o corte o mais rente possível do solo. Os colmos foram então despalhados e tiveram o ponteiro destacado. Em seguida, foram pesados em balança digital tipo gancho, marca Soil Control (precisão = $0,02 \mathrm{~kg}$ ), com capacidade de $50 \mathrm{~kg}$.

Os rendimentos brutos de açúcar e de álcool foram calculados utilizando o valor de quantidade de açúcar bruto determinado por análise tecnológica do caldo, pela amostragem de 10 colmos por tratamento, foram calculados de acordo com metodologia descrita por Caldas (1998):

$$
\mathrm{RAç}=\left(\frac{\mathrm{PCC} * \mathrm{PC}}{100}\right)
$$

Em que: RAç - rendimento em açúcar em $\mathrm{kg}$ $\mathrm{ha}^{-1}$; PCC - quantidade de açúcar bruto em \% contido nos colmos e determinada em laboratório; PC - produção de colmos em kg $\mathrm{ha}^{-1}$.
$\mathrm{RA}=((\mathrm{PCC} * \mathrm{~F})+\mathrm{ARL}) * \mathrm{Fg} * 10 * \mathrm{PC}$

Em que: RA - rendimento de álcool bruto em litro por tonelada de cana; PCC - quantidade de açúcar bruto em \% contido nos colmos e determinada em laboratório; F - fator de transformação estequiométrica de sacarose em uma molécula de glicose mais uma de frutose, igual a 1,052; ARL - são os açúcares redutores livres em $\%$, cujos valores variam de 0,7 a $0,85 \%$, sendo que a destilaria utiliza 0,7 para PCC alto; Fg - fator de Gay Lussac igual a 0,6475; PC - produção de colmos em t ha' .

O teste de normalidade de ShapiroWilk e o teste de homocedasticidade de Breusch-Pagan dos dados, não rejeitou a hipótese de normalidade e de homoscedasticidade dos erros, considerando um nível de significância de 5\%; assim os dados foram submetidos à análise da variância pelo teste $\mathrm{F}(p<0,05)$, e apresentando efeito significativo, foi realizada a análise de regressão polinomial linear e quadrática para os níveis de reposição hídrica. Para o fator aplicação de nitrogênio e ciclo, as médias foram comparadas entre si pelo Teste t à $5 \%$ de significância. Para as análises estatísticas foi utilizado o programa estatístico SISVAR ${ }^{\circledR}$ (FERREIRA, 2011).

\section{RESULTADOS E DISCUSSÃO}

As fases da cana-de-açúcar analisadas com relação à variável $\mathrm{NE}$, apresentaram efeito significativo isoladamente para fase II com relação aos fatores RH e C. Quando se analisa a interação entre estes fatores houve efeito significativo para $\mathrm{RH} \times \mathrm{N}$ x C na fase III. Arantes (2012) obteve resultados quanto ao número de entrenós que correlacionaram positivamente com a produtividade de colmos, sob manejo irrigado e sequeiro. Estes resultados evidenciam a relação direta entre a irrigação por gotejamento subsuperficial e a fase de maturação da cana-de-açúcar para a NE. A variável produtiva rendimento bruto de açúcar (RBAç) apresentou efeito significativo para a interação entre RH x C e RH x N. Estes resultados evidenciaram relação direta entre a 


\section{CRESCIMENTO E RENDIMENTO DA CANA-DE-AÇÚCAR IRRIGADA SUBMETIDA A DIFERENTES REPOSIÇÕES HÍDRIÇAS E NITROGÊNIO}

irrigação por gotejamento subsuperficiale o $\mathrm{N}$ aplicado via irrigação na RBAç da cana-deaçúcar. A variável produtiva rendimento bruto de álcool (RBAl) apresentou efeito significativo para a interação entre $\mathrm{RH} \times \mathrm{C}$ e RH x N. Estes resultados evidenciaram relação direta entre a irrigação por gotejamento subsuperficial e o $\mathrm{N}$ aplicado via irrigação sobre o RBAl da cana-de-açúcar. O efeito significativo do nitrogênio nas variáveis produtivas pode ser atribuído ao fornecimento do fertilizante em pequenas doses ao longo do ciclo de cultivo, aumentando a absorção e favorecendo o aproveitamento do nitrogênio, por apresentar sincronismo maior de disponibilização e absorção de nutrientes para as plantas (GAVA et al., 2011; KÖLLN, 2012). Carvalho et al. (2009) também

A)

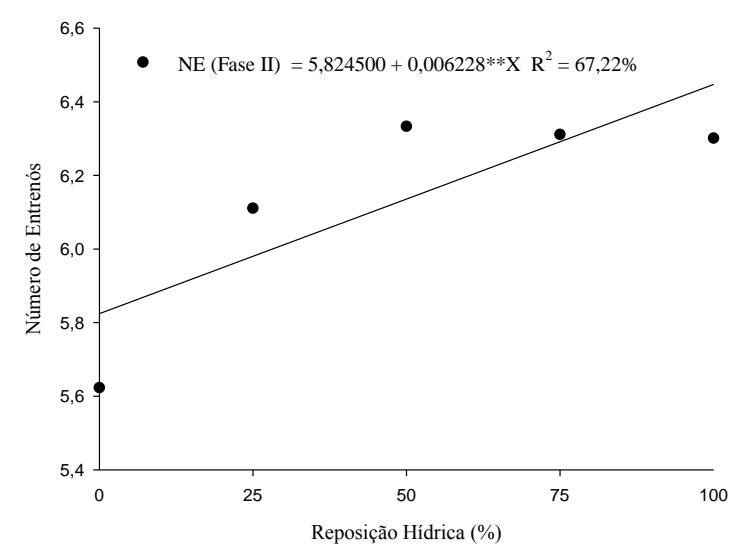

verificaram aumento no rendimento bruto de álcool, com o aumento do nível de irrigação.

$\mathrm{O} \mathrm{NE}$ analisado isoladamente quanto ao fator $\mathrm{C}$ na fase II, apresentou a média superior em $38,62 \%$ para cana-soca quando comparado a cana-planta. A análise do desdobramento de $\mathrm{N}$ dentro de cada nível de RH Ciclo na fase III, apresentou efeito significativo para $0 \% \mathrm{RH}$ na Cana-soca, sendo que a maior média foi observada no tratamento ComN, com o aumento médio de $9,67 \%$ quando comparado a SemN.

Diante dos resultados, estimados para fase II, pode-se verificar um incremento de até $9,64 \%$ para $\mathrm{RH}$ de $100 \%$, quando esta comparada com a ausência de reposição hídrica (Figura 2A).

B)

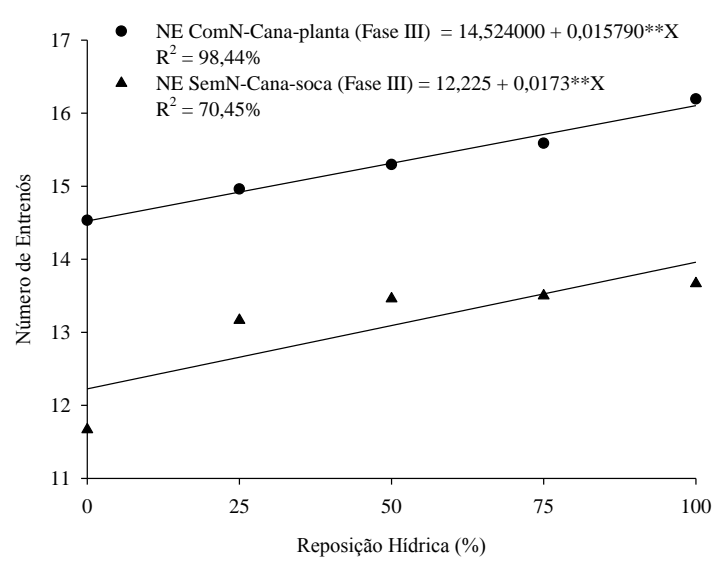

** e * significativo a $1 \%$ e $5 \%$, respectivamente, pelo teste $\mathrm{F}$.

Figura 2. Número de entrenós em função da Reposição Hídrica na Fase II (A) e na Fase III (B) da cana-deaçúcar, Rio Verde, Goiás, safras 2011/2012 e 2012/2013.

A análise do desdobramento de $\mathrm{RH}$ dentro de cada nível de $\mathrm{N}$ Ciclo na fase III, adequaram-se a relações lineares, com $\mathrm{R}^{2}$ de 98,44 e 91,42\% para ComN na Cana-planta, e Sem $\mathrm{N}$ na Cana-soca, indicando que apenas 1,56 e $8,58 \%$ das variações não são explicadas pela variação da RH aplicada na fase III. Mediante os resultados estimados para fase III, pode-se observar que a cada $25 \%$ de $\mathrm{RH}$, houve o incremento de 0,39 , que corresponde respectivamente a $2,45 \%$, totalizando incrementos de até 1,56 , que correspondem respectivamente a $9,8 \%$ para $\mathrm{RH}$ de $100 \%$, conforme Figura 2B. O déficit hídrico também influenciou o número de entrenós, que reduziu esse índice nas plantas submetidas à suspensão da rega fase I, nos dois genótipos avaliados por Machado et al. (2009). O genótipo IACSP 94-2094 apresentou maior número de entrenós do que IACSP 96-2042, independentemente da fase fenológica. Porém, estes mesmos autores recomendam que essa variável deve ser analisada em conjunto com o comprimento de entrenó, que aumentou $(P<0,05)$ em consequência do déficit hídrico de tal forma 
que, quanto mais tardia foi a suspensão da rega, maior foi o comprimento do entrenó.

O crescimento é afetado pelo déficit hídrico por restrições tanto na divisão como no alongamento celular em cana-de-açúcar. Decréscimos na expansão das folhas e colmo ocorrem antes da redução de folhas verdes e, posteriormente, afetam o acúmulo de fitomassa e de sólidos solúveis no caldo
(INMAN-BAMBER, 2004). A análise do desdobramento de Ciclo dentro de cada nível de RH N, na fase III, apresentou efeito significativo em todos os níveis avaliados, sendo que as maiores médias foram observadas em Cana-planta, com o aumento médio de $18,08 \%$ quando comparado a Cana-soca (Tabela 2).

Tabela 2. Resumo da análise do desdobramento de Ciclo dentro de cada nível de RH N na fase III de cultivo da cana-de-açúcar, Rio Verde, Goiás, safras 2011/2012 e 2012/2013.

\begin{tabular}{cccc}
\hline Regime & \multirow{2}{*}{ Nitrogênio } & \multicolumn{2}{c}{ Número de Entrenós (NE) } \\
\cline { 3 - 4 } Hídrico & ComN & $14,5325 \mathrm{a}$ & Cana-soca \\
\hline \multirow{2}{*}{$0 \%$} & SemN & $14,7525 \mathrm{a}$ & $12,9175 \mathrm{~b}$ \\
& ComN & $14,9600 \mathrm{a}$ & $11,6675 \mathrm{~b}$ \\
$25 \%$ & SemN & $15,0675 \mathrm{a}$ & $12,5825 \mathrm{~b}$ \\
& ComN & $15,2950 \mathrm{a}$ & $13,1650 \mathrm{~b}$ \\
$50 \%$ & SemN & $15,2725 \mathrm{a}$ & $12,9975 \mathrm{~b}$ \\
& ComN & $15,5875 \mathrm{a}$ & $13,4600 \mathrm{~b}$ \\
$75 \%$ & SemN & $15,2100 \mathrm{a}$ & $13,0825 \mathrm{~b}$ \\
& ComN & $16,1925 \mathrm{a}$ & $12,3325 \mathrm{~b}$ \\
$100 \%$ & SemN & $15,7525 \mathrm{a}$ & $13,3750 \mathrm{~b}$ \\
& & & $13,6675 \mathrm{~b}$ \\
\hline
\end{tabular}

* Médias seguidas de mesma letra nas linhas não diferem entre si segundo teste Tukey a 5\% de significância.

O desdobramento do RBAç para o fator $\mathrm{RH} \times \mathrm{N}$ se adequou respectivamente a relações lineares, com $\mathrm{R}^{2}$ de 86,93 e $71,85 \%$ para Com $\mathrm{N}$ e Sem $\mathrm{N}$, indicando que apenas 13,07 e $28,15 \%$ das variações não são explicadas pela variação da RH. Mediante os resultados estimados, pode-se observar que ComN, a cada $25 \%$ de RH, houve o incremento de 2,41 t ha ${ }^{-1}$, que correspondem a 25,98\% (Figura
3A). SemN, o aumento máximo ocorreu na RH de $59,34 \%$ com aumentos de até $31,60 \mathrm{Mg}$ $\mathrm{ha}^{-1}$. Dantas Neto et al. (2006) observaram que a adubação de cobertura nas doses de $157 \mathrm{~kg}$ $\mathrm{ha}^{-1}$ de $\mathrm{N}$ mais $148 \mathrm{~kg} \mathrm{ha}^{-1}$ de $\mathrm{K}_{2} \mathrm{O}$, proporcionaram aumentos significativos na qualidade tecnológica da cana-de-açúcar, com acréscimo de 39,8\% para RBAç alcançando um rendimento de $12,58 \mathrm{Mg} \mathrm{ha}^{-1}$ de açúcar.
A)

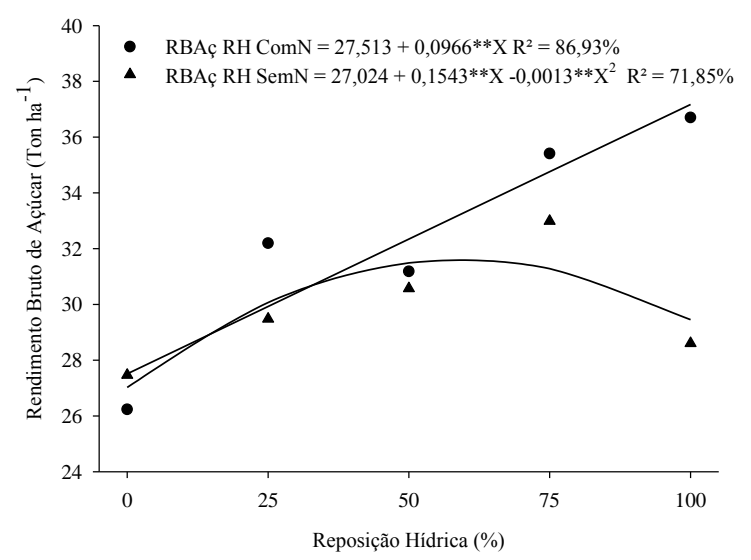

B)

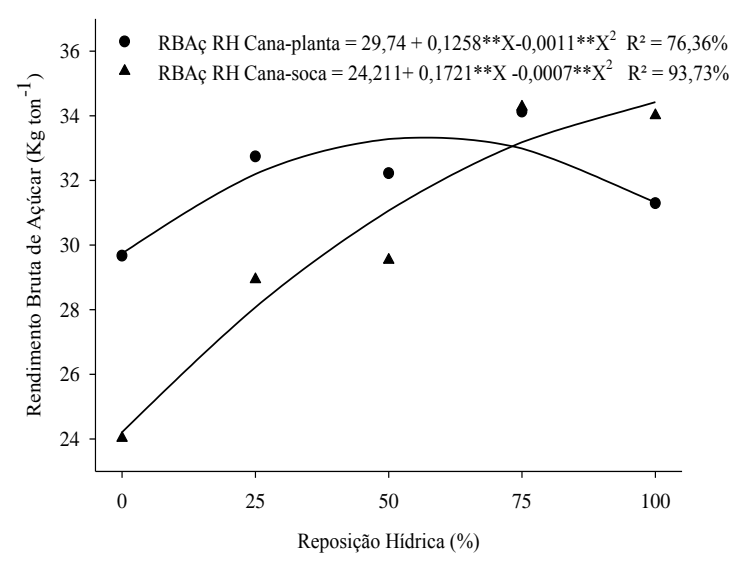

** e * significativo respectivamente a $(P<0,01$ e 0,05$)$ segundo teste $\mathrm{F}$.

Figura 3. Rendimento bruto de açúcar em função da Reposição Hídrica x Nitrogênio (A) e da Reposição Hídrica x Ciclo (B) na cana-de-açúcar, Rio Verde, Goiás, safras 2011/2012 e 2012/2013. 
O desdobramento do RBAç para o fator $\mathrm{RH} \times \mathrm{C}$ se adequou respectivamente a relações quadráticas, com $\mathrm{R}^{2}$ de 76,36 e $93,73 \%$ para cana-planta e cana-soca. Mediante os resultados estimados, pode-se observar que o ponto de máximo para cana-planta e cana-soca ocorreram respectivamente com 57,18 e $100 \%$ de $\mathrm{RH}$, atingindo valores de até 33,33 e 34,42 t ha $^{-1}$ (Figura 3B).

O desdobramento do RBAl para o fator $\mathrm{RH} \times \mathrm{N}$ se adequou respectivamente a relações

A)

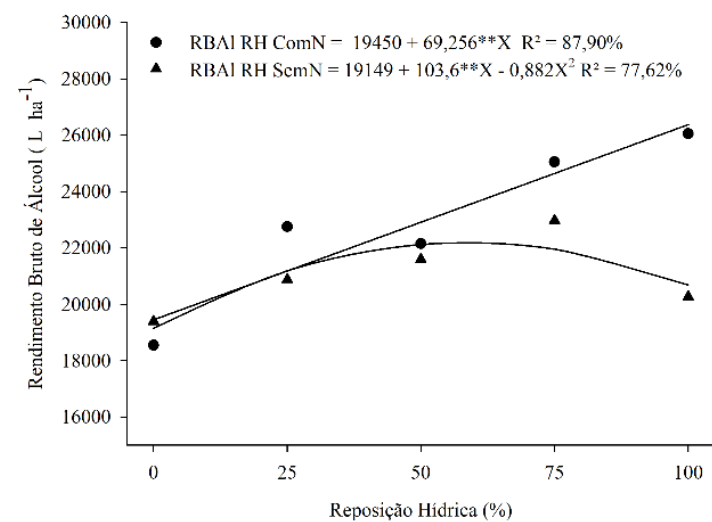

** $\mathrm{e}^{*}$ significativo respectivamente a $(\mathrm{p}<0,01$ e 0,05$)$ segundo teste $\mathrm{F}$.

Figura 4. Rendimento bruto de álcool em função da Reposição Hídrica x Nitrogênio (A) e da Reposição Hídrica x Ciclo (B) na cana-de-açúcar, Rio Verde, Goiás, safras 2011/2012 e 2012/2013.

O desdobramento do RBAl para o fator RH x C se adequou aos modelos quadrático e linear, com $\mathrm{R}^{2}$ de 71,02 e 89,89\% para canaplanta e cana-soca, respectivamente. Pode-se observar que na cana-soca, a cada $25 \%$ de $\mathrm{RH}$, houve o incremento de $1.789,15 \mathrm{~L} \mathrm{ha}^{-1}$, que correspondem a 28,77\% (Figura 4B). Para a Cana-planta, o aumento máximo ocorreu na RH de 60,34\%, atingindo valores de até 23.960,12 $\mathrm{L} \mathrm{ha}^{-1}$.

Dantas Neto et al. (2006) observaram que a adubação de cobertura, nas doses de 157 $\mathrm{kg} \mathrm{ha}{ }^{-1}$ de $\mathrm{N}$ mais $148 \mathrm{~kg} \mathrm{ha}^{-1}$ de $\mathrm{K}_{2} \mathrm{O}$, proporcionaram aumentos significativos na qualidade tecnológica da cana-de-açúcar, com

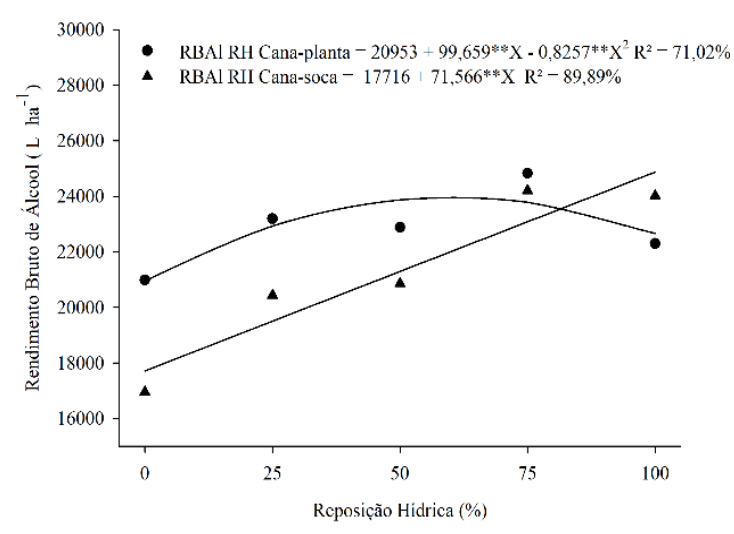

lineares e quadrática, com $\mathrm{R}^{2}$ de 87,90 e $77,62 \%$ para ComN e SemN, indicando que apenas 12,10 e $22,38 \%$ das variações não são explicadas pela variação da RH. Mediante os resultados estimados, pode-se observar que no ciclo de cana-planta a cada $25 \%$ de $\mathrm{RH}$, houve o incremento de $1.731,4 \mathrm{~L} \mathrm{ha}^{-1}$, que correspondem a $26,25 \%$, conforme a Figura 4A. Já para SemN, o aumento máximo ocorreu na $\mathrm{RH}$ de $58,73 \%$, atingindo valores de até 22.353,53 $\mathrm{L} \mathrm{ha}^{-1}$.

B)

acréscimo de $42,2 \%$ para $\mathrm{RBAl}$, alcançando um rendimento de $8,91 \mathrm{~L} \mathrm{ha}^{-1}$ de álcool.

\section{CONCLUSÕES}

O aumento da reposição hídrica combinada com adubação nitrogenada proporciona incrementos de aproximadamente $10 \%$ no número de entrenós da cana-deaçúcar.

O máximo rendimento de açúcar e de álcool $\left(31,60 \mathrm{Mg} \mathrm{ha}^{-1}\right.$ e 22.353,53 $\left.\mathrm{L} \mathrm{ha}^{-1}\right)$ da cana-de-açúcar sem a aplicação de nitrogênio, é verificado na reposição hídrica de $60 \%$. 
A reposição hídrica combinada com a adubação nitrogenada promoveu aumento linear do rendimento bruto de açúcar e de álcool.

\section{AGRADECIMENTOS}

Os autores agradecem ao Conselho Nacional de Desenvolvimento Científico e Tecnológico (CNPq); à Coordenação de Aperfeiçoamento de Pessoal de Nível Superior (CAPES); à Fundação de Amparo à Pesquisa do Estado de Goiás (FAPEG) e ao Instituo Federal Goiano - campus Rio Verde, pelo apoio financeiro e estrutural.

\section{REFERÊNCIAS BIBLIOGRÁFICAS}

ALLEN, R. G.; PEREIRA L. S.; RAES, D. Crop evapotranspiration. Rome, 297 p. (FAO Irrigation and Drenage Paper, 56). 1998.

ARANTES, M. T. Potencial produtivo de cultivares de cana-de-açúcar sob os manejos irrigado e sequeiro. 2012. 65p. Dissertação (Mestrado em Agronomia) UNESP.

BARBOSA, F. S. Resistência à seca em cana-de-açúcar para diferentes níveis de disponibilidade hídrica no solo. 2010. 81p. Dissertação (Mestrado) - Escola Superior de Agricultura "Luiz de Queiroz". Piracicaba.

\section{CALDAS, C. Manual de análises selecionadas para indústrias sucroalcooleiras. Maceió: Sindicato da Indústria e do Álcool do Estado de Alagoas, 424p, 1988.}

CARVALHO, C. M. de; AZEVEDO, H. M. de; DANTAS NETO, J.; FARIAS, C. H. de A.; SILVA, C. T. S. da; GOMES FILHO, R. R. Rendimento de açúcar e álcool da cana -de -açúcar submetida a diferentes níveis de irrigação. Revista Brasileira de Ciências Agrárias, v. 4, p. 72-77, 2009.
DANTAS NETO, J; FIGUEREDO, J. L. C; FARIAS, C. H. A; AZEVEDO, H. M; AZEVEDO, C. A. V. Resposta da cana-deaçúcar, primeira soca, a níveis de irrigação e adubação de cobertura. Revista Brasileira de Engenharia Agrícola e Ambiental, v.10, n.2, p.283-288, 2006.

DIOLA, V.; SANTOS, F. Fisiologia. In: SANTOS, F.; BORÉM, A.; CALDAS, C. (Eds.). Cana-de-açúcar: bioenergia, açúcar e etanol - tecnologias e perspectivas. 2 ed. Viçosa: Os Editores, 2012. p. 25-49.

EMBRAPA. Sistema brasileiro de classificação de solos. 3. ed. Rio de Janeiro: Embrapa Informação Tecnológica, 2013. 353 p.

FARIAS, C. H. A.; FERNANDES, P. D.; GHEYI, H. R.; DANTAS NETO, J. Qualidade industrial de cana-de-açúcar sob irrigação e adubação com zinco, em Tabuleiro Costeiro paraibano. Revista Brasileira de Engenharia Agrícola e Ambiental, v. 13, n.4, 419-428, 2009.

FERREIRA, D. F. Sisvar: a computerstatisticalanalysis system. Ciência e Agrotecnologia, v. 35, n.6, p. 1039-1042, 2011.

GAVA, G. J. DE C.; SILVA, M. DE A.; SILVA, R. C. DA; JERONIMO, E. M.; CRUZ, J. C. S.; KÖLLN, O. T. Produtividade de três cultivares de cana-de-açúcar sob manejos de sequeiro e irrigado por gotejamento. Revista Brasileira de Engenharia Agrícola e Ambiental, v.15, n.3, p.250-255, 2011.

GOUVEIA NETO, C. G. Rendimento agroindustrial da cana-de-açúcar sob suplementação hídrica e parcelamento de nitrogênio. 145p. 2012. Tese (Doutorado). Universidade Federal de Campina Grande Campina Grande, 2012.

GOUVÊA, J. R. F. Mudanças climáticas e a expectativa de seus impactos na cultura da cana-de-açúcar na região de Piracicaba, SP. 
2008. 98 p. Dissertações (Mestrado) - Escola Superior de Agricultura Luiz de Queiroz. Piracicaba, 2008.

INMAN-BAMBER, N. G. Sugarcane water stress criteria for irrigation and drying off. Field Crops research, Australia, v. 89, p.107$122,2004$.

KÖLLN, O. T. Interação entre os estresses de nitrogênio e disponibilidade hídrica no fracionamento isotópico de ${ }^{13} \mathrm{C}$ e na produtividade em soqueira de cana-deaçúcar. 2012. 104 f. Dissertação (Mestrado em Ciências). Centro de Energia Nuclear na Agricultura, Universidade de São Paulo, Piracicaba, 2012.

KÖPPEN, W.; GEIGER, R. Klimate der Erde. Gotha: Verlag Justus Perthes. 1928.

MACHADO, R. S.; RIBEIRO, R. V.; MARCHIORI, P. E. R.; MACHADO, D. F. S. P.; MACHADO, E. C.; LANDELL, M. G. A. Respostas biométricas e fisiológicas ao déficit hídrico em cana-de-açúcar em diferentes fases fenológicas. Pesquisa agropecuária brasileira, v.44, n.12, p.1575-1582, dez. 2009.

MASCHIO, R. Produtividade da água em biomassa e energia para 24 variedades de cana-de-açúcar.
2011. 87p. Dissertação (Mestrado) Escola Superior de Agricultura "Luiz de Queiroz".

OLIVEIRA, E. C. A.; FREIRE, F. J.; OLIVEIRA, A. C.; NETO, D. E. S.; ROCHA, A. CARVAlHO, T.; L. A. Produtividade, eficiência de uso da água e qualidade tecnológica de cana-de-açúcar submetida a diferentes regimes hídricos. Pesquisa agropecuária brasileirav.46, n.6, p.617-625, jun. 2011.

ROSSETTO, R. Maturação da cana-deaçúcar. Disponível em: $<$ http://www.agencia.cnptia.embrapa.br/gestor /cana-deacucar/arvore/CONTAG01_90_22122006154 841.html>. Acessado em:10 Out. 2013.

SOUSA, D. M. G.; LOBATO, E. Cerrado: Correção do solo e adubação. 2.ed. Brasília, Embrapa Informação Tecnológica, 2004. 416p.

TAIZ, L.; ZEIGER, E. Fisiologia vegetal. 3ed. Porto Alegre: Artmed, 2004. 700-719 p.

THORNTHWAITE, C. W.; MATHER, J. R. The water balance. Centerton: Drexel Institute of Technology, Laboratory of Climatology, 104 p. (Publications in Climatology, v. 8, n. 1), 1955. 